\title{
Implantação de uma Arquitetura de Software para Monitoramento de Dados Ambientais em um Cenário de Smart Campus
}

\author{
Haelton Carvalho ${ }^{1}$, Joiner Sá ${ }^{1}$, Fabricio Farias ${ }^{1}$ \\ ${ }^{1}$ Universidade Federal do Pará (UFPA) - Cametá - Pará - Brasil \\ haelton@ufpa.br, joinersa@hotmail.com, fabriciosf@ufpa.br
}

\begin{abstract}
This article presents a software project for future deployment at Federal University of Pará, Cametá campus. In this project, an Internet of Things (IoT) network will be implemented for real-time and environmental monitoring using temperature, CO2, CO, humidity and C4H10 sensors. For this, the Dojot ecosystem was installed to receive data in real time and an IoT Agent was implemented for data collection. In order to present the results, the data were organized on average per minute to present the general environmental conditions of the area under monitoring.
\end{abstract}

Resumo. Este artigo apresenta um projeto de software para futura implantação no campus Cametá da Universidade Federal do Pará. No projeto, será implementada uma rede de Internet das Coisas (IoT) para monitoramento ambiental e em tempo real usando os sensores de temperatura, CO2, CO, Umidade e C4H10. Para isso, foram instalados o ecossistema Dojot para recebimento de dados em tempo real e um IoT Agent para coleta de dados. Para apresentação dos resultados, os dados foram organizados em média por minuto para apresentação das condições ambientais gerais da área sob monitoramento.

\section{Introdução}

A Internet das Coisas (IoT) tem por finalidade interconectar dispositivos ou objetos utilizados no dia a dia, através da Internet, e assim podendo influenciar na qualidade de vida e decisões de uma sociedade [Zhamanov 2017]. Nesta perspectiva, surgiu a ideia de Smart Campus, onde, utilizando redes de dispositivos IoT torna-se possível receber e enviar dados do mundo real que serão usados em decisões para diferentes tipos de necessidades dentro de um Campus Universitário.

Para monitorar características ambientais em tempo real em um Smart Campus, é necessário desenvolver uma arquitetura genérica capaz de dar suporte aos dispositivos instalados no campo via uma comunicação composta por hardware e software, isto é, utilizando uma rede IoT [Pinto et al. 2020]. Desta forma, destacamos o ecossistema Dojot é uma proposta brasileira e open source que tem como objetivo facilitar a comunicação e armazenamentos de dados em tempo real a partir da IoT [Dojot 2021].

Diante do contexto, este trabalho pretende propor uma infraestrutura de software para a coleta de dados de sensores ambientais utilizando a plataforma brasileira Dojot. Os dados coletados são meteorológicos e de mitigação de Gases do Efeito Estufa (GEE), sendo eles: temperatura, umidade, $\mathrm{CO}, \mathrm{CO} 2$, e C4H10. Para a realização dos testes, foi implementado um IoT Agent responsável em enviar os dados para a Dojot. 
Nas seções seguintes serão descritos o cenário de aplicação (Seção 2), a arquitetura do sistema e protótipo (Seção 3). Ao final, serão discutidos resultados (Seção 4) e considerações finais (Seção 5).

\section{Cenário de Aplicação}

A infraestrutura foi instalada na Universidade Federal do Pará, Campus Cametá (Smart Campus), sendo composta por um servidor instalado nas dependências da Divisão de Tecnologia da Informação (DTI), equipado com Processador Quad Core com 4 núcleos e $2.9 \mathrm{GHz}$ por núcleo, $6 \mathrm{~GB}$ de memória RAM e $120 \mathrm{~GB}$ de armazenamento. Para a comunicação sem fio entre os equipamentos/sensores e servidor serão instalados um gateway e sete endnodes dispostos na área externa do Campus, conforme a Figura 1.

Na comunicação, será utilizada a modulação Long Range (LoRA) [Vega et al. 2019]. No planejamento, os endnodes serão instalados com distância máxima de 100 metros em relação ao gateway, sendo a distância definida por Ugarte (2019) como uma distância eficaz para a tecnologia LoRA. Para a instalação dos sensores, foram selecionados os prédios e as áreas verdes do Campus, assim, sendo possível monitorar os dados ambientais em áreas de concreto e arborizadas. Por fim, para a coleta dos dados, cada endnode será conectado a um Arduino que, por sua vez, estará recebendo os dados dos sensores ambientais e estabelecendo comunicação com o nó Gateway.

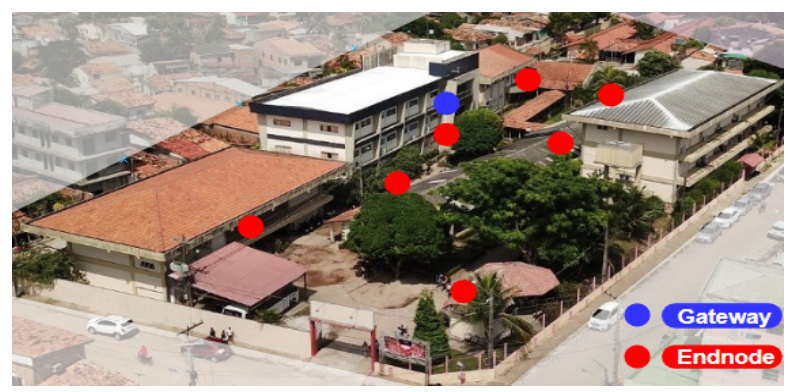

Figura 1. Cenário de Medição - Campus Cametá.

\section{Arquitetura do Sistema e Protótipo}

Na Figura 2, é ilustrada a arquitetura de software implementada neste trabalho. Para esse estudo foi utilizado um IoT Agent, representado por um script que simula os equipamentos instalados no Campus. O IoT Agent foi adotado para a realização de testes e validação da comunicação com a Dojot. Para a troca de informação foi instalado o protocolo Message Queuing Telemetry Transport (MQTT) [Pinto et al. 2020]. Além disso, foi implantado o orquestrador Kubernetes [Kubernetes 2021] junto com a Dojot. Por fim, foi instalado o Balance [Nginx 2021] para divisão da carga de trabalho entre os nós dos containers, assim evitando a sobrecarga do sistema. 


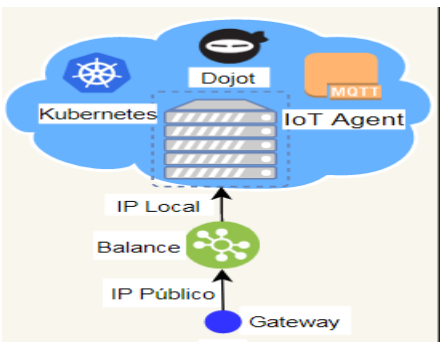

(a)

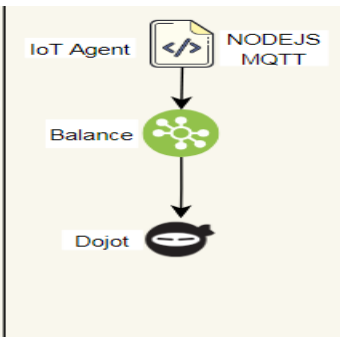

(b)

Figura 2. Arquitetura de software do Sistema proposto. (a) Estrutura em nuvem. (b) Fluxo dos dados coletados.

Para esse trabalho foi implementada a arquitetura de rede em nuvem, vide Figura 2 (a). Os dados coletados pelo IoT Agent são transmitidos até a Dojot a partir do Balance, conforme ilustrado na Figura 2 (b). Para organizar os dados coletados pelos sensores, uma lista de variáveis foi criada, conforme a Tabela 1. O Sistema Internacional de Unidades (SI) foi utilizado como padrão de medidas e em alguns casos foi necessário incluir uma forma de obtenção da variável, ou seja, se a variável foi obtida diretamente na coleta ou se deverá ser calculada após a medição.

Para os valores mínimo e máximo das variáveis, foram calculadas a média dos dados históricos meteorológicos no município de Cametá no ano de 2020 [INMET 2021] e os resultados das análises de emissões de GEE disponibilizados pelo INMETRO [INMETRO 2021].

Tabela 1. Lista de Variáveis Coletadas no Experimento.

\begin{tabular}{|l|c|c|c|l|}
\hline \multicolumn{1}{|c|}{ Nome da Variável } & Intervalo entre as Amostras & Unidade* & Formato do Gráfico & \multicolumn{1}{c|}{ Fórmula } \\
\hline Temperatura & 10 minutos & $\mathrm{K}$ & Linhas & ${ }^{\circ} \mathrm{C}=\mathrm{K}-273,15$ \\
\hline Umidade & 30 minutos & $\%$ & Linhas & $U R=\frac{W}{W_{s}} \times 100 \%$ \\
\hline CO2 (Dióxido de Carbono) & 20 minutos & PPM ou \% & Linhas & $\rho(t, p)=\rho(25 \circ C, 1013 h P a) x \frac{p}{1013} \times \frac{298}{(273+t)}$ \\
\hline CO (Monóxido de Carbono) & 20 minutos & PPM ou \% & Linhas & $\rho(t, p)=\rho(25 \circ C, 1013 h P a) x \frac{p}{1013} \times \frac{298}{(273+t)}$ \\
\hline & & & & \\
C4H10 (Gás Butano) & 20 minutos & PPM ou \% & Linhas & $\rho(t, p)=\rho(25 \circ C, 1013 h P a) x \frac{p}{1013} x \frac{298}{(273+t)}$ \\
\hline
\end{tabular}

\section{Resultados}

Nesta seção, são apresentados os resultados obtidos e armazenados na Dojot, assim como, também é apresentada a média calculada a partir dos dados de temperatura, umidade, $\mathrm{CO}, \mathrm{CO} 2$, e C4H10 fornecidos pelo IoT Agent. Na Figura 3 (a), são ilustrados os dados de um endnode organizados na Dojot.

Já na Figura 3 (b), é ilustrada a média dos dados coletados a partir da Dojot. Esse resultado demonstra que é possível estimar outros resultados a partir de dados ambientais coletados a partir da implantação do Smart Campus. Deste modo, demonstrando o potencial positivo do monitoramento a partir da implantação desta infraestrutura na Universidade.

Os resultados também demonstraram que a arquitetura em nuvem é viável para a comunicação, recepção, armazenamento e fácil manutenção de dados coletados a partir 
de dispositivos instalados em campo. Deste modo, podendo permitir a obtenção de dados ambientais a partir de uma rede IoT.

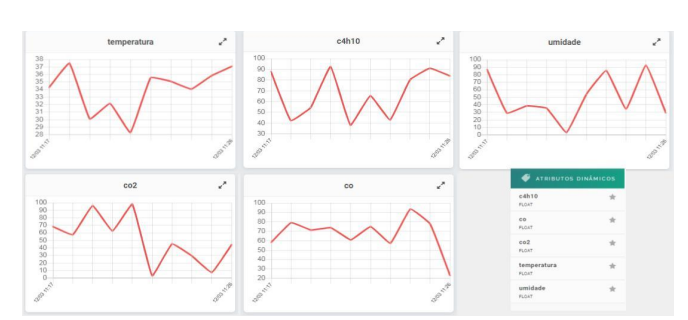

(a)

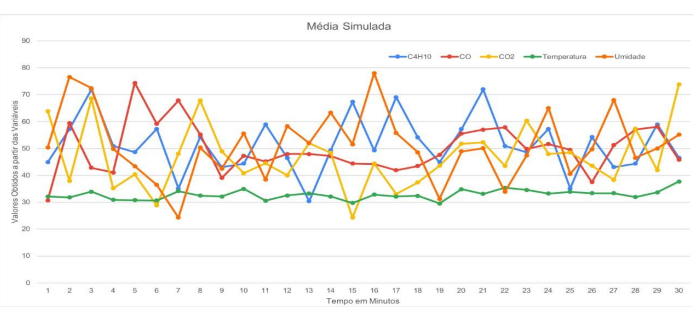

(b)

\section{Figura 3. (a) Acesso aos dados retornados da Dojot via dashboard. (b) Média Simulada a partir das variáveis.}

\section{Considerações Finais}

Esse trabalho apresentou uma solução baseada em software para monitoramento em tempo real das condições ambientais de um Campus Universitário. Os gráficos ilustram os resultados da obtenção dos dados a partir da configuração do software e da média de dados ambientais obtidas e coletadas durante um período de tempo.

A partir dos resultados é possível concluir que a arquitetura de software composta pela Dojot, Kubernetes e Balance é uma alternativa satisfatória para a implantação de um Smart Campus. Nos próximos trabalhos, o IoT Agent será incrementado a partir da implantação de equipamentos físicos instalados no campo. Para isso será adotada uma infraestrutura física conectada via comunicação sem fio na frequência de $915 \mathrm{MHz}$, baseada em endnodes e gateways do tipo LoRA.

\section{Referências}

Dojot (2021), http://www.dojot.com.br/, Março.

INMET (2021) “Dados Históricos”, https://portal.inmet.gov.br/dadoshistoricos, Março.

INMETRO (2021), "Desenvolve Projetos de Analises de Gases”, https://www.gov.br/inmetro, Março.

Kubernetes (2021), https://kubernetes.io/, Junho.

Nginx (2021), https://www.nginx.com/, Junho.

Pinto, K. S. et al. (2020) "Um Estudo de Caso de Internet das Coisas Utilizando o Ecossistema Dojot: Da Configuração a Utilização", Conferência Nacional em Comunicações, Redes e Segurança da Informação, X Edição, p. 95.

Ugarte, L. F. et al. (2019) "LoRa Communication as a Solution for Real-Time Monitoring of IoT Devices at UNICAMP”, In: IEEE International Conference on Smart Energy Systems and Technologies (SEST), p.1-6.

Vega, L. F. U., Garcia, Maique Corrêa, O. Rocheti, Enrico, PENTEADO LACUSTA JUNIOR, Eduardo, Pereira, Leandro S., ALMEIDA, M. C. (2019) "LoRa Communication as a Solution for Real-Time Monitoring of IoT Devices at UNICAMP", 2 IEEE International Conference on Smart Energy Systems and Technologies (SEST), pp.1-6, Porto, Portugal.

Zhamanov, Azamat et al. (2017) "IoT Smart Campus Review and Implementation of IoT Applications into Education Process of University", 13th International Conference on Electronics, Computer and Computation (ICECCO), IEEE. p. 1-4. 